Acta Cryst. (2002). A58 (Supplement), C190

STRUCTURE-REACTIVITY RELATIONSHIPS OF INCLUSION COMPOUNDS

$\underline{\text { L.R. Nassimbeni }}$

University of Cape Town Chemistry Rondebosch CAPE TOWN 7701 SOUTH AFRICA

An understanding of the reactivities of solids depends on our knowledge of the strengths and directions of the ionic and non-covalent interactions, which hold the structure together, and will permit us to predict the behavior of the solid under differing thermodynamic conditions.

Our laboratory has concentrated on the study of inclusion compounds with a view to understanding their various properties and how these are related to their structures. We have synthesised a wide range of host-guest compounds and characterised them by measuring their thermal stabilities and analysing their kinetics of formation and desorption:

$$
\operatorname{Host}_{(\mathrm{s}, \alpha)}+\text { n Guest }_{(\mathrm{g})} \underset{\mathrm{k}_{\mathrm{r}}}{\mathrm{\textrm {k } _ { \mathrm { f } }}}=\text { Host } \bullet(\text { Guest })_{\mathrm{n}(\mathrm{s}, \beta)}
$$

for the forward, enclathration reaction, we have noted that a threshold pressure of the guest vapour, $\mathrm{p}_{0}$, is required to start the reaction. This phenomenon gives rise to anti-Arrhenius behaviour, in that for a given pressure $\mathrm{p}$, the reaction is slower at higher temperature.

The reserve, desorption reaction, is typically carried out by isothermal thermal gravimetry, and allows us to enclathrate activation energies for the process.

Keywords: STRUCTURE，THERMODYNAMICS，KINETICS packing of molecules in the solid state. This will allow us to compute the

Acta Cryst. (2002). A58 (Supplement), C190

\section{NEUTRON DIFFRACTION FROM HIGH-TEMPERATURE SUPERCONDUCTORS AND COLOSSAL MAGNETORESISTIVE MATERIALS}

J. Jorgensen

Argonne National Laboratory Materials Science Division Building 223 ARGONNE IL 60439 USA

Structural probes are critical to our understanding of materials whose properties derive from strong interactions among electronic structure, magnetic ordering, and crystal structure. The two most striking examples of recent years are high-temperature superconductors and colossal magnetoresistive materials. In both cases, structural studies have had two objectives - to guide the design of materials with optimized physical properties by establishing how chemical composition and crystal structure control the behavior and to provide fundamental information needed to understand the underlying physics. Impressive success has been achieved in both areas. for example, we understand the structural features that will give rise to the highest $T_{c}$ in the layered cuprate superconductors and we know how to maximize the temperature of colossal magnetoresistive behavior in manganite compounds by adjusting cation size and average $M n$ valence. Studies leading to this understanding have revealed a beautifully complex behavior. In the layered cuprate high-temperature superconductors subtle ordering of oxygen vacancies and the competition between $\mathrm{CuO}_{2}$ plane buckling and superconductivity can have a remarkable effect on properties. In the manganites, competition among available states of charge ordering and magnetic ordering can lead to complex phase separation. In both systems, the most recent work has revealed the importance of short-range self-organization of charge and spin - for example, the so-called striped phases, polarons, etc. Understanding these inhomogeneous states has presented new challenges to structural studies, resulting in increasing use of small-angle and diffuse scattering.

This work is support by the US Dept. of Energy, Basic Energy Sciences Materials Sciences, under contract No. W-31-109-ENG-38.

\section{Keywords: NEUTRON DIFFRACTION SUPERCONDUCTIVITY COLOSSAL MAGNETORESISTIVE MATERIALS}

\author{
Acta Cryst. (2002). A58 (Supplement), C190

\section{ELECTRON CRYSTALLOGRAPHY} \\ D. L. Dorset \\ ExxonMobil Research \& Engineering Co., Annandale, NJ, USA
}

T.K. Sixma ${ }^{1}$ K. Brejc ${ }^{1}$ P. Celie ${ }^{1}$ W.J. van Dijk ${ }^{1}$ R. V. Klaassen ${ }^{2}$ J. van der Oost $^{2}$ M. Schuurmans ${ }^{2}$ A.B. Smit ${ }^{2}$

${ }^{1}$ Division of Molecular Carcinogenesis, Netherlands Cancer Institute, Plesmanlaan 121, 1066 CX Amsterdam, The Netherlands 2 Department of Molecular and Cellular Neurobiology, Research Institute Neurosciences Vrije Universiteit, Faculty of Biology, De Boelelaan 1087, 1081 HV Amsterdam, The Netherlands

The first neurotransmitter receptors to be described were the nicotinic acetylcholine receptors. These pentamers of homologous subunits have served as prototypes for ligand-binding ion channels and their complicated ligandbinding and ion-channel properties have been described in exquisite detail over the past 40 years. Based on the beautiful electron microscopy work of Nigel Unwin they have also long been among the best-described membrane protein structures. Detailed atomic coordinates however, have been slow in coming. The water-soluble acetylcholine binding protein (AChBP) from the snail Lymnaea stagnalis is a homologue of the ligand binding domains of nicotinic receptors with very similar pharmacology. Based on the crystal structure of AChBP we can explain the biochemical data on the pentameric ligand-gated ion channels in three-dimensions. The ligand-binding site is found at the interface of two subunits, consisting of a 'cage' of mostly hydrophobic or aromatic residues provided by six different regions in the two subunits. Charge complementarity with the quaternary ammonium in the ligands such as acetylcholine or nicotine takes place by cation- $\pi$ interactions. Based on modeling studies and ligand-binding analysis we will discuss this important drug-target in some detail.

\section{Keywords: ION CHANNEL PROTEIN STRUCTURE} ACETYLCHOLINE
Electron diffraction overcomes the challenges to structure analysis imposed by the microcrystalline state by removing the reflection overlaps found in powder diffraction data. Actual use of intensity data for $a b$ initio quantitative structure analysis (electron crystallography), however, has remained a controversial topic, owing to strong multiple scattering perturbations. In 1975, conventional direct phasing methods were first applied successfully to experimental electron diffraction amplitudes. Unknown crystal structures were determined later and refined by Fourier techniques. Often, the success of direct methods in electron crystallography need not demand an approximation to kinematical scattering. The successful evaluation of three-phase invariants depends on the information content of the Patterson function. An adequate representation of interatomic vectors in the Patterson map, sufficiently overlapping the ideal crystal autocorrelation function, can remain even if multiple scattering obscures the Rfactor fit to experimental structure factor amplitudes. This explains the surprising application of electron crystallography to important inorganic problems (high $\mathrm{T}_{\mathrm{c}}$ superconductors, zeolites, metal catalysts). Nevertheless, a conscious effort must be made to improve data quality by optimized specimen preparation, use of low electron wavelength, and perhaps precession methods to record true integrated intensities. The major current challenge to $a b$ initio determinations seems to be structure refinement, where constraints to local minima must always be imposed, if a kinematical model is followed. Dynamical refinements have been proposed in addition to the current technique of perfecting the initial electron crystallographic model by Rietveld refinement against powder $\mathrm{x}$-ray data.

Keywords: ELECTRON DIFFRACTION CRYSTAL STRUCTURE DETERMINATION DIRECT METHODS 